\title{
Neuromyelitis optica mimics the morphology of spinal cord tumors
}

\author{
İlknur Erol ${ }^{1}$, Murat Özkale², Tülin Savaş ${ }^{1}$ Özlem Alkan³ ${ }^{3}$, Melih Çekinmez ${ }^{4}$, Ayșe Erbay ${ }^{5}$ \\ Divisions of ${ }^{1}$ Pediatric Neurology, and ${ }^{5}$ Pediatric Hematology and Oncology, ${ }^{2}$ Department of Pediatrics, ${ }^{3}$ Department of \\ Radiology, and ${ }^{4}$ Department Neurosurgery, Başkent University Faculty of Medicine, Adana, Turkey. \\ Email: ilknur_erol@yahoo.com
}

Received: $2 \overline{7}$ July 2015, Revised: 17 September 2015, Accepted: 23 October 2015

\begin{abstract}
SUMMARY; Erol İ, Özkale M, Savaş T, Alkan Ö, Çekinmez M, Erbay A. Neuromyelitis optica mimics the morphology of spinal cord tumors. Turk J Pediatr 2016; 58: 309-314.

Neuromyelitis optica (NMO) is an autoimmune disorder of the central nervous system, that predominantly affects the spinal cord and the optic nerve. Its key features include transverse myelitis, commonly associated with extensive inflammation spanning three or more consecutive vertebral segments. Longitudinal extensive spinal cord lesions can also occur in systemic autoimmune diseases, infections, vascular and metabolic disorders, subsequent to irradiation, intramedullary tumors and paraneoplastic myelopathies. We present a case study of an 8-year-old girl seropositive for antibodies against the aquaporin 4 who displayed longitudinal extensive spinal cord lesions, that was initially misdiagnosed as an intramedullary tumor.
\end{abstract}

Key words: neuromyelitis optica, intramedullary tumor, longitudinally extensive spinal cord lesions, transverse myelitis.

Neuromyelitis optica (NMO), also known as Devic's disease, is a chronic inflammatory disease of the central nervous system (CNS) that is associated with severe bilateral optic neuritis accompanied by transversemyelit is. ${ }^{1}$ Neuromyelitis optica spectrum disorders (NMOSD) include various forms of this condition ranging from wide spread disturbances in brain function to more limited pathology such as isolated transverse myelitis or optic neuritis. ${ }^{2}$ Typically, disease onset is either acute or subacute, and the symptoms are likely to persist when not treated appropriately. The most common forms of NMOSD include optic neuritis and transverse myelitis. While the former can lead to decreased or complete loss of vision, the latter is characterized by extensive inflammatory injury of the spinal cord spanning three or more consecutive vertebral segments. ${ }^{1,2}$ Diagnosis and monitoring of NMOSD is most commonly done using magnetic resonance imaging (MRI). In addition, examination of visual evoked potentials and analysis of the cerebrospinal fluid (CSF) are also helpful in ensuring accurate diagnosis. ${ }^{1,3}$ The aquaporin 4 serum autoantibody, also known as NMO-IgG, is a specific biomarker for NMOSD. Several studies have reported that serum anti-aquaporin4 titers have been correlated with clinical disease activity, drop after immunosuppressive treatment, and remain low during remissions. Even using the most sensitive assays, 12 percent of patients with a clinical diagnosis of NMO or NMOSD are seronegative for NMO-IgG. ${ }^{4,5}$ During the acute phase of the disease, the inflamed lesions enlarge progressively, secondary to tissue edema. ${ }^{3}$ Such morphology of the spinal cord is often misdiagnosed initially as an intramedullary tumor. ${ }^{6,7}$

To the best of our knowledge, only one pediatric case of NMO misdiagnosed as spinal cord tumor has been reported and discussed in literature. ${ }^{8}$ Here, we present a child who displayed longitudinally extensive spinal cord lesions resulting in a "swollen spinal cord" appearance, that was initially misdiagnosed as an intramedullary tumor.

\section{Case Report}

An eight-year-old female patient, who was diagnosed provisionally with a cervical 
intramedullary tumor and who underwent continuous monitoring in the neurosurgical intensive care, was referred to our department when she displayed symptoms like agitation and confusion. One month prior to admission in our hospital, the patient had complained of abdominal pain, following which she underwent appendectomy in another hospital. She started to display progressive weakness in both of her lower extremities 3 weeks before admission. An intramedullary tumor was detected in the spinal cord MRI, following which she was sent to the neurosurgery department for further evaluation, including a spinal cord biopsy, in order to confirm the presence of the tumor. Further examination revealed that the patient was afebrile, and displayed neither a rash nor a meningismus. However, a slight dysfunction in mental processing, without speech disturbance, was noted. The strength of her lower extremities was scored at 1/5. Deep tendon reflexes were found to be hyperactive in both lower extremities but were normal in the upper extremities. Plantar reflexes with clonus were found in both of the lower extremities. A sensory examination revealed loss of sensitivity to light touch, temperature, and pinprick at approximately $\mathrm{C}_{4}-\mathrm{T}_{4}$ spinal levels. The patient was also diagnosed with bladder dysfunction. The patient underwent again brain and whole spine MRI which revealed a long segment of spinal cord enlargement with high signal intensity in the center, surrounded by rostral cysts, poorly marginated tissue mass and poorly marginated enhancement at the $\mathrm{C}_{2}-\mathrm{T}_{4}$ level (Figs. 1A and 1B). Based on these observations, the patient was first diagnosed with spinal tumor and concomitant paraneoplastic limbic encephalitis. To treat this condition, intravenous dexamethasone treatment was started. To describe the pathology, neurosurgeons performed a partial C6 and total C7-T1 laminectomy. After opening the dura mater during surgery, they observed

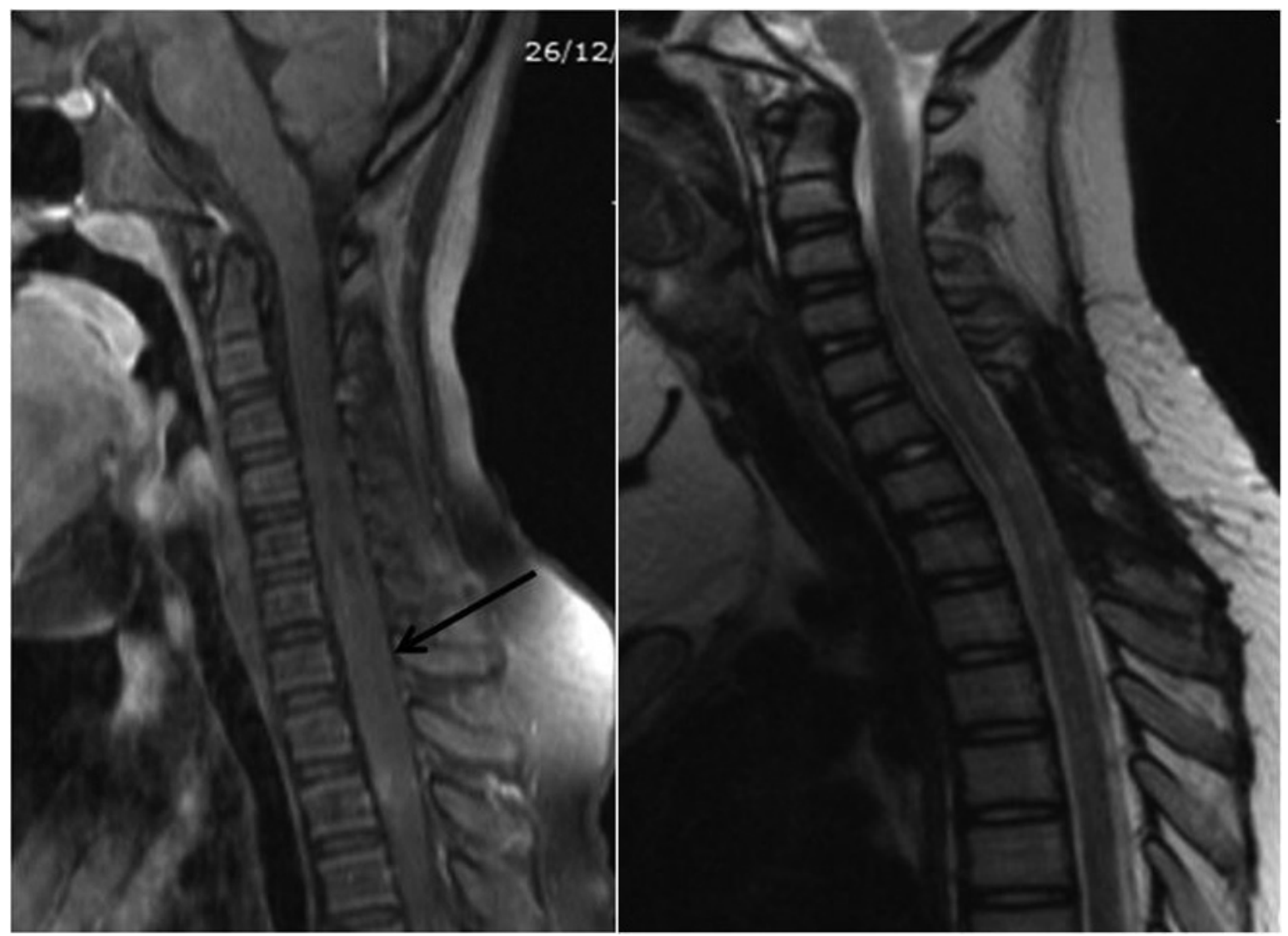

Fig. 1A-1B. Sagittal T2-weighted image (A) and post contrast sagittal T1-weighted image with fat saturation (B) show a long segment of spinal cord enlargement with high signal intensity in the center, surrounded by adjacent rostral cysts and patchily enhancement. 
that the spinal cord, although edematous, appeared otherwise normal. They obtained needle biopsy for frozen section examination that showed only signs of gliosis. Following these results, the patient was reevaluated and transferred to the child neurology department, where she was diagnosed with long segment transverse myelitis. An extensive examination was conducted in order to identify potential etiologies of an inflammatory pseudotumor. The results of laboratory examinations such as complete blood cell count, liver enzymes, kidney function parameters, C-reactive protein and erythrocyte sedimentation rate were evaluated, and found to be normal. Serological assays for autoimmune diseases and connective tissue diseases, such as anticardiolipin, antimicrosomal, antinuclear andanti-dsDNA antibodies were all negative. Serological assays for Lyme disease, viral infections such as Epstein Barr virus (EBV), cytomegalovirus and Herpes Simplex virus were also negative. Based on the results of the MRI and additional clinical examination, the patient was diagnosed with NMOSD. From the day she was admitted to our clinic, she was administered an intravenous methylprednisolone pulse therapy $(30 \mathrm{mg} / \mathrm{kg} /$ day for 7 days), followed by oral prednisolone ( $1 \mathrm{mg} / \mathrm{kg}$ per day). Once the steroid therapy was initiated, the patient continued to display a gradual clinical improvement in condition, and was able to walk by day 30 of her illness. Although her visual acuity, fundoscopic examination and visual evoked potential studies were normal. On day 34 of admission, the patient tested positive for

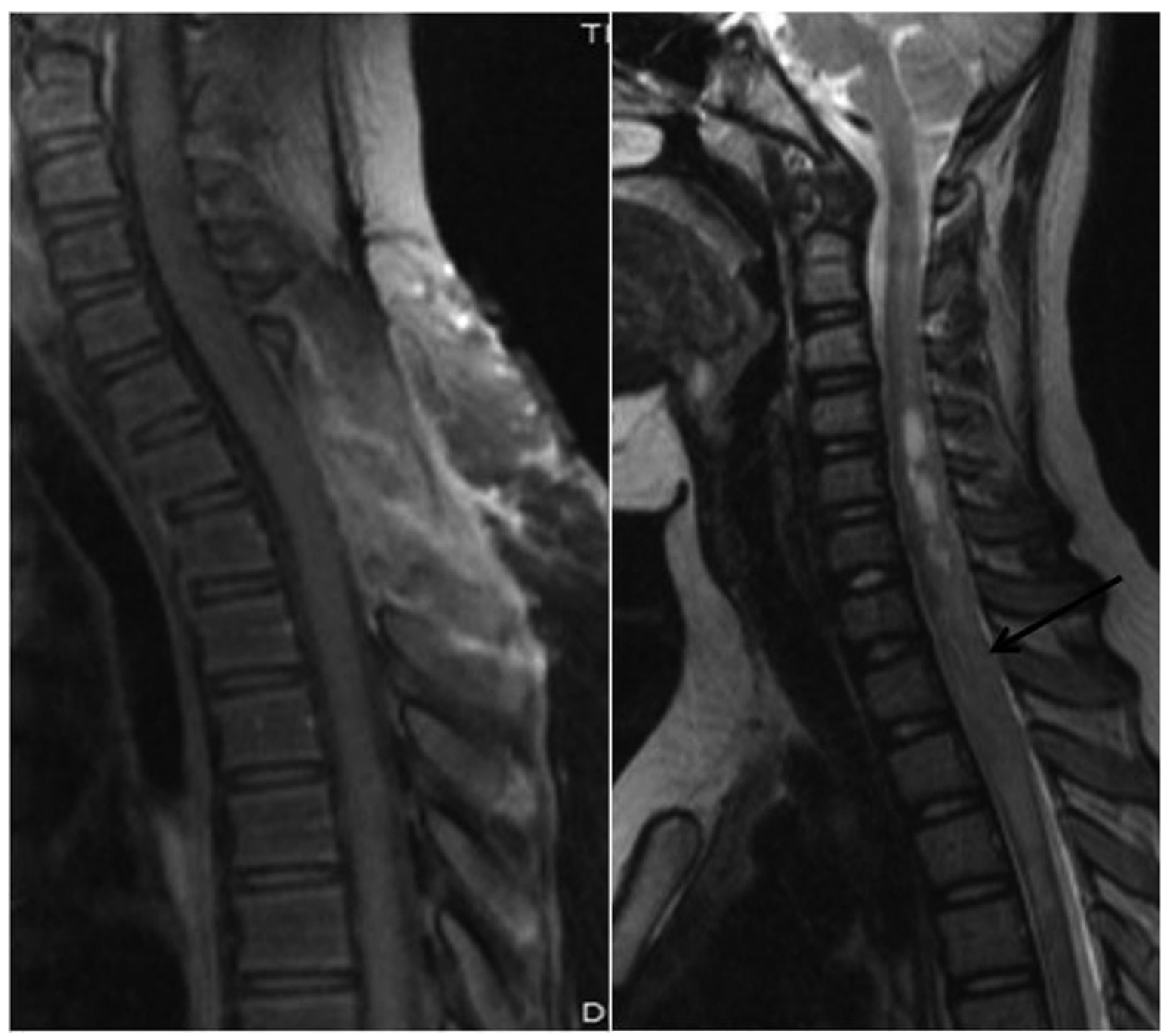

Fig. 2A-2B. Follow-up MRI scans show regression of spinal cord expansion and loss of contrast enhancement. 
aquaporin IgG antibodies (NMO-IgG). After we obtained these results, oral azathioprine (2.5 $\mathrm{mg} / \mathrm{kg}$ per day) was added to prednisolone (1 $\mathrm{mg} / \mathrm{kg}$ per day) treatment. During a 1-month follow-up examination, an MRI was performed, which revealed a significant regression of the lesions. (Figs. 2A and 2B). The patient was regularly followed up at the out-patient clinic for at least 4 weeks after being discharged from the hospital until preparation of this report. A neurological examination performed 3 months after admission, showed complete recovery to normal neurologic function. After 3 months, the prednisolone dosage was tapered, while treatment with oral azathioprine was continued.

\section{Discussion}

Longitudinally extensive spinal cord lesions are known to occur during several disease conditions such as systemic autoimmune diseases, infections, vascular and metabolic disorders, subsequent to irradiation, intramedullary tumors and paraneoplastic myelopathies. ${ }^{1,2,6-10} \mathrm{On}$ a transverse plane, spinal cord masses can be classified into the following: 1. intramedullary, the mass of tissue within the spinal cord parenchyma; 2 . intradural-extramedullary, the mass between the spinal cord and the dura mater; 3 . extradural, the mass outside the dura mater but within the bony spine. ${ }^{11}$ Intramedullary lesions are most commonly found in astrocytomas, ependymomas and gangliogliomas, but occur less frequently in hemangioblastomas or during metastases. ${ }^{12}$ In MRI studies, spinal ependymomas appear sharply marginated, with homogenously enhancing masses, and slightly hyperintense on T2-weighted images. This enhancing mass, on average, extends over approximately four vertebral segments. Also, ependymomas are commonly (up to $90 \%$ ) associated with adjacent rostral or caudal cysts. ${ }^{13}$ Since our patient displayed longitudinally extensive mass like spinal cord lesions with adjacent rostral cysts and poorly marginated tissue mass enhancement, together with disrupted neurological function but without physical brain lesions on the MRI, she was first diagnosed with an intramedullary tumor and paraneoplastic limbic encephalitis. Consistent with this case, there are a few case reports in literature where longitudinally extensive spinal cord lesions resulting in a 'swollen spinal cord' appearance have been misdiagnosed as spinal cord intramedullary tumors. ${ }^{6-8}$ The MRI findings of NMO was characterized by longitudinally extensive spinal cord lesions (3 vertebral segments) that preferentially involve spinal central gray matter which are $\mathrm{T} 2$ hyperintense and frequently $\mathrm{T} 1$ hypointense. Additionally these lesions may acutely expand the cord and exhibit patchy enhancement throughout their length. On the other hand sometimes heterogeneous contrast enhancement and cystic areas may also occur on intramedullary tumor lesions. ${ }^{14}$

Thus far, less than 10 cases of NMO misdiagnosed as spinal cord tumor have been reported. Only one of these, reported by Horbinski et $\mathrm{al}^{8}$., is a pediatric case that was orginally diagnosed as an intramedullary tumor of the spinal cord. They reported the case of a 10-year-old child with NMO, whose MRI and intraoperative findings were strongly suggestive of a neoplastic process. The patient was operated upon, resulting in gross total resection of the tumor. However, subsequent pathological examination revealed an inflammatory demyelinating lesion. Since serological examination indicated that the patient carried NMO-IgG antibodies, she was diagnosed with NMO. Similarly our patient had also undergone spinal cord biopsy. Since pathological examination failed to yield specific findings, several diagnostic possibilities were considered, including NMOSD, multiple sclerosis (MS), systemic lupus erythematosus (SLE) and numerous infectious pathogens. An extensive diagnostic examination was performed in this patient in order to exclude a majority of the abovementioned conditions.

Neuromyelitis optica is an autoimmune disorder of the CNS, that predominantly affects the spinal cord and the optic nerves. In 1999, Wingerchuk et al. ${ }^{15}$ proposed diagnostic criteria for NMO, that included three absolute requirements: 1. optic neuritis; 2 . acute myelitis; and, 3 . lack of pathological symptoms in other CNS regions. However, these criteria failed to identify patients whose symptoms surpassed the spinal cord and the optic system, or those whose clinical course was consistent with NMO but was accompanied by brain lesions as seen on MRI scans. The discovery of circulating IgG1 antibodies against the astrocytic water 
channel aquaporin 4 (NMO-IgG) in NMO patients, and the evidence that NMO-IgGs are involved in the development of neuromyelitis optica, revolutionized our understanding of the disease. ${ }^{16}$ Subsequently, a set of revised criteria were proposed to enable accurate diagnosis of NMO. These included the presence of, 1. optic neuritis; 2 . myelitis; and 3 . at least two out of three supportive criteria (MRI evidence of a contiguous spinal cord lesion in $\geq 3$ segments, brain MRI not diagnostic of MS, and NMO-IgGseropositivity). ${ }^{1}$ Recently, the clinical spectrum of NMO was extended to include patients who display only optic neuritis or long segment transverse myelitis, and such conditions were included under NMOSD. ${ }^{2}$ In addition to these findings, a group of children with NMO have brain involvement at presentation associated with clinical features of encephalopathy, seizures, and/or lesions on brain MRI. ${ }^{17,18}$ Since our patient had agitation and confusion without lesions on brain MRI, she can also be classified under NMOSD.

Immunotherapy of NMO can be administered in two parts: 1 . rescue therapy of an acute disease relapse; and 2. disease-modifying therapy. Corticosteroids and plasma exchange are the most commonly used therapeutic strategies in rescue therapy. The effects of both treatment modalities are rapid and significant improvements can be detected within days of their initiation. ${ }^{19,20}$ Our patient displayed gradual improvement once she was started on a rescue therapy with corticosteroids. The most common disease-modifying therapy includes orally administered drugs like azathioprine or mycophenolate mofetil, or parenteral drugs like rituximab. ${ }^{16,21}$ In one of the largest retrospective studies conducted (including 99 patients), it was reported that azathioprine decreased the annual relapse rate by $76 \%$ and either improved or stabilized disability in $40 \%$ patients during a 12 -month period. ${ }^{22}$ Since our patient was diagnosed as seropositive for NMOSD during the very first occurrence of long segment transverse myelitis, she was treated with azathioprine at a dosage of 2.5 $\mathrm{mg} / \mathrm{kg} /$ day, together with prednisone which was administered in a prolonged tapering regimen from $1 \mathrm{mg} / \mathrm{kg}$ to $0.25 \mathrm{mg} / \mathrm{kg}$ over a 3 -month period. The patient continues to remain on a daily azathioprine treatment, and has shown no relapse during a 5-month-follow up period.
In conclusion, spinal cord lesions resulting from neuromyelitis can be misdiagnosed as an intramedullary tumor. Neuromyelitis and intramedullary tumor can not be distinguished with only radiological findings. But the presence of cystic areas, hypointensity in T1-weighted images and the extension of the cervical lesion to the brain stem may suggest NMO. ${ }^{14}$ Early diagnosis and treatment of NMO is essential for an accurate prognosis and determination of sequelae. Our findings emphasize the importance of testing for NMO-IgGs in patients diagnosed with intramedullary tumors in the spinal cord although 12 percent of patients with a clinical diagnosis of NMO or NMOSD are seronegative for NMO-IgG. We believe that in lieu of the possible adverse side-effects of spinal cord biopsy procedures, testing for NMO-IgGs is justified in patients where the presence/absence of spinal cord tumors cannot be determined conclusively.

\section{REFERENCES}

1. Wingerchuk DM, Lennon VA, Pittock SJ, Lucchinetti CF, Weinshenker BG. Revised diagnostic criteria for neuromyelitis optica. Neurology 2006; 66: 1485-1489.

2. Wingerchuk DM, Lennon VA, Lucchinetti CF, Pittock SJ, Weinshenker BG. Thespectrum of neuromyelitis optica. Lancet Neurol 2007; 6: 805-815.

3. O'Riordan JI, Gallagher HL,Thompson AJ et al. Clinical, CSF, and MRI findings in Devic's neuromyelitis optica. J Neurol Neurosurg Psychiatry 1996; 60: 382-387.

4. Wingerchuk DM, Banwell B, Bennett JL, et al. International consensus diagnostic criteria for neuromyelitis optica spectrum disorders. Neurology 2015; 85: 177-189.

5. Jiao Y, Fryer JP, Lennon VA, et al. Updated estimate of AQP4-IgG serostatus and disability outcome in neuromyelitis optica. Neurology 2013; 81: 1197-1204.

6. Habek M, Adamec I, Brinar VV. Spinal cord tumor versus transverse myelitis. Spine J 2011; 1: 1143-1145.

7. Brinar M, Rados M, Habek M, Poser CM. Enlargement of the spinal cord: inflammation or neoplasm? Clin Neurol Neurosurg 2006; 108: 284-289.

8. Horbinski C, Pollack IF, Wiley C, Murdoch G. A 10year old girl with neck pain. Brain Pathol 2010; 20: 519-522.

9. Kitley JL, Leite MI, George JS, et al. The differential diagnosis of longitudinally extensive transverse myelitis. Mult Scler 2011; 18: 271-285.

10. Trebst C, Raab P, Voss EV, et al. Longitudinal extensive transverse myelitis-it's not all neuromyelitis optica. Nat Rev Neurol 2011; 7: 688-698.

11. Bourgouin PM, Lesage J, Fontaine S, et al. A pattern approach to the differential diagnosis of intramedullary spinal cord lesions on MR imaging. AJR Am J 
Roentgenol 1998; 170: 1645-1649.

12. Lowe GM. Magnetic resonance imaging of intramedullary spinal cord tumors. J Neurooncol 2000; 47: 195-210.

13. Sun B, Wang C, Wang J, et al. MRI features of intramedullary spinal cord ependymomas. J Neuroimaging 2003; 13: 346-351.

14. Barnett Y, Sutton IJ, Ghadiri M, et al. Conventional and Advanced Imaging in Neuromyelitis Optica. AJNR Am J Neuroradiol 2014; 35: 1458-1466.

15. Wingerchuk DM, Hogancamp WF, Hogancamp WF, Obrien PC, Weinshenker BG. The clinical course of neuromyelitis optica (Devic's syndrome). Neurology 1999; 53: 1107-1114.

16. Lennon VA, Kryzer TJ, Pittock SJ, Verkma AS, Hinson SR. IgG marker of optic spinal multiple sclerosis binds to the aquaporin-4 water channel. J Exp Med 2005; 202: 473-477.

17. McKeon A, Lennon VA, Lotze T, et al. CNS aquaporin-4 autoimmunity in children. Neurology. 2008; 71: 93100.
18. Banwell B, Tenembaum S, Lennon VA, et al. Neuromyelitis optica-IgG in childhood inflammatory demyelinating CNS disorders. Neurology 2008; 70: 344-352.

19. Wingerchuk DM, Weinshenker BG. Acute disseminated encephalomyelitis, transverse myelitis, and neuromyelitis optica. Continuum (Minneap Minn) 2013; 19: 944-967.

20. Palace J, Leite MI, Nairne A, Vincent A. Interferon Beta treatment in neuromyelitis optica: increase in relapses and aquaporin 4 antibody titers. Arch Neurol 2010; 67: 1016-1017.

21. Scott TF, Frohman EM, De Seze J, et al. Evidencebased guideline: clinical evaluation and treatment of transverse myelitis: report of the Therapeutics and Technology Assessment Subcommittee of the American Academy of Neurology. Neurology 2011; 77: 2128-2134.

22. Costanzi C, Matiello M, Lucchinetti CF, et al. Azathioprine: tolerability, efficacy, and predictors of benefit in neuromyelitis optica. Neurology 2011; 77: 659-666. 